\title{
Little evidence for the use of diode lasers as an adjunct to non-surgical periodontal therapy
}

\author{
Abstracted from \\ Slot DE, Jorritsma KH, Cobb CM, Van der Weijden FA. \\ The effect of the thermal diode laser (wavelength $808-980 \mathrm{~nm}$ ) in non-surgical periodontal therapy: \\ a systematic review and meta-analysis. J Clin Periodontol 2014; 41: 681-692. \\ Address for correspondence: Dagmar Else Slot, Department of Periodontology, \\ Academic Centre for Dentistry Amsterdam (ACTA), University of Amsterdam and VU University Amsterdam, \\ Gustav Mahlerlaan 3004, 1081 LA Amsterdam, The Netherlands. E-mail: d.slot@acta.nl
}

\section{Question: How effective is fluoride varnish in preventing dental caries?}

Data sources Medline, PubMed, the Cochrane Central Register of Controlled Trials (CENTRAL) and Embase databases.

Study selection Randomised controlled trials (RCTs) using thermal diode lasers as an adjunct to non-surgical conventional periodontal initial therapy conducted in patients $\geq 18$ years old written in English or Dutch were considered.

Data extraction and synthesis Study assessment data extraction and quality assessment was carried out independently by two reviewers. The main outcome variables were probing pocket depth (PPD) and clinical attachment loss (CAL), but plaque scores (PS), bleeding scores (BS) and the Gingival Index (GI) were also considered. Meta-analysis was carried out using a random effects model.

Results Nine studies involving 247 patients were included. Seven studies were of split mouth design and two were parallel group studies. The study designs showed considerable heterogeneity and follow up ranged from six weeks to six months. Meta-analysis found no significant effect on PPD, CAL and PS. There was however a significant effect for $\mathrm{Gl}$ and BS favouring adjunctive use of the diode laser Conclusions The collective evidence regarding adjunctive use of the diode laser with SRP indicates that the combined treatment provides an effect comparable to that of SRP alone. With respect to BS the results showed a small but significant effect favouring the diode laser, however, the clinical relevance of this difference remains uncertainStandard. This systematic review questions the adjunctive use of diode laser with traditional mechanical modalities of periodontal therapy in patients with periodontitis. The strength of the recommendation for the adjunctive use of the diode laser is considered to be 'moderate' for changes in PPD and CAL.

\section{Commentary}

This article presents a rigorous and high-quality systematic review of the current literature looking at the adjunctive use of the diode laser after scaling and root planing in the treatment of periodontal disease. The clinical question was appropriately focused:

Based on randomised controlled clinical trials, what is the effect of the adjunctive use of a diode laser following non-surgical periodontal debridement (SRP) during initial phase of periodontal therapy on the clinical parameters of periodontal inflammation, ie probing pocket depth, clinical attachment level, plaque score, bleeding score and gingival index, compared to SRP alone?

Standard methods were used in this systematic analysis, giving it a high degree of credibility. The results of this systematic review indicate that the adjunctive use of the diode laser after SRP is unwarranted. With consistently beneficial results not being demonstrable, and with the addition of thermal risk to the periodontal tissues being a reality in such applications, ethical questions arise regarding whether appropriate risk/benefit ratios are present.

Further, these results are similar to those reported for other laser wavelengths used intrasulcularly as an adjunctive procedure to SRP, such as the Nd:YAG, for both 'Nd- YAG Laser Curettage' in the early years and more recently 'LANAP' (Laser Assisted New Attachment Procedure). The rationale for both procedures, and also the diode laser protocols investigated in this article, wrongly accepts the notion that the removal of sulcular epithelium is necessary in the first place. The need for the removal of sulcular epithelium in the nonsurgical treatment of periodontal disease was long-ago discredited by numerous studies, ${ }^{1}$ and in fact, more recently the treatment code for curettage has been removed from the ADA's CDT- 4 code listing, indicating that the profession as a whole believes that subgingival curettage, by whatever method, is a treatment with no clinical value. ${ }^{2}$

With consistently beneficial results from adjunctively using the diode (and Nd-YAG) laser not being demonstrated, it then becomes moot to speculate on potential mechanisms of claimed benefits by proponents of this technique.

\section{Practice points}

- There is no substitute for meticulous root debridement, no matter how boring, tedious and difficult this might be to accomplish. The success of periodontal therapy is directly related to the quality of root debridement

- Technology that promotes better root debridement (ie flap surgery when indicated) offers more potential for improving periodontal outcomes than technology focused on soft tissue debridement of the sulcular epithelium, as the removal of sulcular epithelium has been convincingly shown to be unnecessary.

Douglas N Dederich

School of Dentistry, Faculty of Medicine and Dentistry, University of Alberta, Edmonton, Canada

1. Dederich DN, Drury GI. Laser curettage: where do we stand? J Calif Dent Assoc 2002; 30: 376-382.

2. ADA.org: ADA Statement on Lasers in Dentistry, 2009

Evidence-Based Dentistry (2015) 16, 16. doi:10.1038/sj.ebd.6401078 